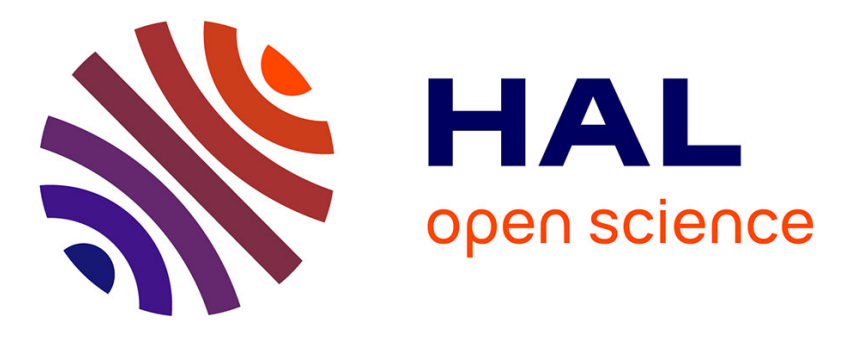

\title{
Early asymmetric inter-hemispheric transfer in the auditory network: insights from infants with corpus callosum agenesis
}

Parvaneh Adibpour, Jessica Dubois, Marie Laure Moutard, Ghislaine Dehaene-Lambertz

\section{To cite this version:}

Parvaneh Adibpour, Jessica Dubois, Marie Laure Moutard, Ghislaine Dehaene-Lambertz. Early asymmetric inter-hemispheric transfer in the auditory network: insights from infants with corpus callosum agenesis. Brain Structure and Function, 2018, 223 (6), pp.2893-2905. 10.1007/s00429-018-1667-4 . hal-02004827

\section{HAL Id: hal-02004827 \\ https://hal.science/hal-02004827}

Submitted on 2 Feb 2019

HAL is a multi-disciplinary open access archive for the deposit and dissemination of scientific research documents, whether they are published or not. The documents may come from teaching and research institutions in France or abroad, or from public or private research centers.
L'archive ouverte pluridisciplinaire HAL, est destinée au dépôt et à la diffusion de documents scientifiques de niveau recherche, publiés ou non, émanant des établissements d'enseignement et de recherche français ou étrangers, des laboratoires publics ou privés. 


\section{Early asymmetric inter-hemispheric transfer in the auditory network: insights from infants with corpus callosum agenesis}

Parvaneh Adibpour $\mathrm{MSc}^{\mathrm{a}} *$, Jessica Dubois $\mathrm{PhD}^{\mathrm{a}}$, Marie-Laure Moutard $\mathrm{MD}^{\mathrm{b}}$, Ghislaine Dehaene-Lambertz MD-PhD ${ }^{\text {a }}$

Affiliations :

a. INSERM, UMR 992 ; CEA, NeuroSpin Center; University Paris Saclay, Gif-sur-Yvette, France

b. Assistance-Publique Hôpitaux de Paris, Neuropediatrics Department, Paris, France

Corresponding address

Parvaneh Adibpour

CEA/SAC/DRF/ NeuroSpin/Cognitive Neuroimaging Unit U992

Bât 145, point courrier 156

91191 Gif-sur-Yvette, France

Email: p.adibpour@gmail.com

Phone: +3316908 8172

Fax: +33169087973

Running title: Role of the callosal pathway in the human infant auditory network

\section{Abstract}

The left hemisphere specialization for language is a well-established asymmetry in the human brain. Structural and functional asymmetries are observed as early as the prenatal period suggesting genetically determined differences between both hemispheres. The corpus callosum is a large tract connecting mostly homologous areas; some have proposed that it might participate in an enhancement of the left-hemispheric advantage to process speech. To investigate its role in early development, we compared 13 3-4-month-old infants with an agenesis of the corpus callosum ("AgCC") with 18 typical infants using high-density electroencephalography (EEG) in an auditory task. We recorded event-related potentials (ERP) for speech stimuli (syllables and babbling noise), presented binaurally (same syllable in both ears), monaurally (babbling noise in one ear) and dichotically (syllable in one ear and babbling noise in the other ear). In response to these stimuli, both groups developed an anterior positivity synchronous with a posterior negativity, yet the topography significantly differed between groups likely due to the atypical gyration of the medial surface in AgCC. In particular, the anterior positivity was lateral in AgCC infants while it covered the midline in typical infants. We then measured the latencies of the main auditory response ( $\mathrm{P} 2$ at this age) for the different conditions on the symmetrical left and right clusters. The main difference between groups was a $\sim 60 \mathrm{~ms}$ delay in typical infants relative to $\mathrm{AgCC}$, for the ipsilateral response (i.e. left hemisphere) to babbling noise presented in the left ear, whereas no difference was observed in the case of right-ear stimulation. We suggest that our results highlight an asymmetrical callosal connectivity favoring the right-to-left hemisphere direction in typical infants. This asymmetry, similar to recent descriptions in adults, might contribute to an enhancement of left lateralization for language processing beyond the initial cortical left-hemisphere advantage. 


\section{Keywords}

Corpus callosum, Corpus callosum agenesis, Brain development, electroencephalography EEG, Auditory network, Asymmetry, Inter-hemispheric transfer, Language, Brain specialization

\section{Introduction:}

The left hemisphere specialization for language processing is a well-established example of hemispheric lateralization in the human brain consistently demonstrated in adult studies over the years both at the structural and functional levels (Toga \& Thompson, 2003; Van Essen, 2005). In fetuses and preterm neonates, hemispheric asymmetries are already clearly visible, especially in perisylvian areas. At the macrostructural level, the Yakovlean torque that raises and pushes the right hemisphere forward relative to the left is observed before term age featuring the common characteristic of the human brain: right frontal and left occipital petalia, a more ventral and horizontal left than right Sylvian fissure, a larger left than right planum temporale, and a deeper right than left superior temporal sulcus (Dubois et al., 2010; Dubois et al., 2015; Glasel et al., 2011; Habas et al., 2012; Leroy et al., 2011). Inter-hemispheric differences are also observed at the microstructural level and in the maturational calendar of the superior temporal and inferior frontal regions (Leroy et al., 2011), and of the arcuate fasciculus (Dubois et al., 2009; 2016). Finally, several functional magnetic resonance imaging (fMRI) and near infra-red spectroscopy (NIRS) studies have reported larger activations to speech in the left than the right planum temporale, and more generally in the left than right hemisphere during the first post-natal months (Dehaene-Lambertz, Dehaene, \& Hertz-Pannier, 2002; Mahmoudzadeh et al., 2013; Pena et al., 2003; Shultz, Vouloumanos, Bennett, \& Pelphrey, 2014).

Thus, the left-hemispheric functional advantage for speech processing described in adults appears to be rooted in the first stages of development, suggesting a strong genetic component. The strength of this left-lateralization for speech is difficult to compare between infants and adults. Nevertheless, the functional reorganization after a lesion of the left hemisphere suggests a better plasticity at an early age: linguistic functions may move to the contralateral right hemisphere with less subsequent deficits in infants than later on, suggesting a strengthening of the hemispheric specialization for speech with age (Bates, Vicari, \& Trauner, 1999; DehaeneLambertz, Pena, Christophe, \& Landrieu, 2004). Thus, other factors beyond the initial lefthemispheric bias might contribute to speech lateralization. The corpus callosum, which gathers most of the white matter fibers connecting both hemispheres (Aboitiz, Scheibel, Fisher, \& Zaidel, 1992), has been suggested as a candidate factor (Jeeves \& Temple, 1987; Karbe, Herholz, Halber, \& Heiss, 1998; Selnes, 1974). Caminiti et al. (2009) reported a relative stability of the corpus callosum's structure over the course of evolution between chimps and humans despite the increase in brain size and thus distances between brain areas. They hypothesized that the "relative" slowdown of the inter-hemispheric transfer between humans and chimps incites each hemisphere to specialize. Other authors have postulated a more active role of callosal fibers either through inhibition from one hemisphere to the other (Cook, 1984; Dennis, 1981; Karbe et al., 1998), or excitation (Yazgan et al., 1995).

The growth of fibers constituting the corpus callosum starts during the second trimester of pregnancy, and all callosal connections are in place toward the end of gestation. This tract is heterogenous, constituted of different types of fibers connecting mostly, but not always, homologous areas (Innocenti, 1986). During infancy, pruning of irrelevant and useless fibers occurs (Kostović \& Jovanov-Milošević, 2006), and myelination progresses until the end of 
adolescence, accelerating the inter-hemispheric transfer time (IHTT) (Brody, Kinney, Kloman, \& Gilles, 1987; Yakovlev. \& Lecours., 1967). This IHTT is variable along the tract, depending on the diameter of the fibers, on their state of maturation, and on the distance the signal travels between two connected areas (Ringo, Doty, Demeter, \& Simard, 1994). In adults, the visual IHTT is the slowest (Caminiti, Ghaziri, Galuske, Hof, \& Innocenti, 2009), but given the fast maturation of visual areas during the first post-natal trimester, myelination might compensate for the longer distance between visual areas at this age relative to the closer but less mature auditory areas. The question of when these fibers are sufficiently mature to be functionally efficient is still unknown. Neonates are able to transfer tactile and haptic information from one hand to the other (Sann \& Streri, 2007), suggesting that somatosensory inter-hemispheric connections are rapidly efficient. By contrast, in the visual domain, de Schonen and Mathievet (1990) did not find any signature of visual inter-hemispheric transfer at 10 months due to infants' difficulties in generalizing learning from one visual hemifield to the other (de Schonen \& Mathivet, 1990). They further postulated that visual integration between both hemifields does not emerge before 24 months (Liégeois, Bentejac, \& de Schonen, 2000). However, in a recent study using eventrelated potentials (ERPs) during the first post-natal semester, we were able to measure the IHTT of the visual P1 for faces presented in the left and right hemifields (Adibpour, Dubois, \& Dehaene-Lambertz, 2018). The IHTT decreased from $\sim 315 \mathrm{~ms}$ to $\sim 80 \mathrm{~ms}$. While we related the IHT speed with the maturation of visual fibers from the corpus callosum splenium, we also showed that the transfer of face-specific responses was far from being complete in the first postnatal semester. Regarding the auditory domain, whether inter-hemispheric connectivity is efficient enough to convey speech information during early infancy remains an open question so far.

One way to investigate the role of callosal fibers in the development of inter-hemispheric transfer is to compare typical infants and infants with impaired growth of the corpus callosum. In fact, early development of callosal fibers can be disrupted due to a genetic disease (Bedeschi et al., 2006; Bonneau et al., 2002), environmental factors (Evrard et al., 2003), or no identified causes (Paul et al., 2007), and a partial or complete absence (agenesis) of the corpus callosum (AgCC) might occur with no other brain malformations (isolated agenesis). With a prevalence of at least 1/4000 births (Guillem et al., 2003), this pathology often has little impact on general cognitive abilities (Chiarello, 1980) but may sometimes coincide with a range of specific cognitive impairments. Particularly in the language domain, AgCC subjects may have difficulties in phonological and rhyming processing, syntax and linguistic pragmatics (Sanders, 1989; Temple \& Ilsleya, 1993; Temple, Jeeves, \& Vilarroya, 1989).

So far, studies in adults with AgCC have favored a weak modulation of the linguistic left hemispheric advantage by the corpus callosum. Using fMRI, Pelletier et al. (2011) found similar lateralization indices in six $\mathrm{AgCC}$ adults relative to controls in expressive and receptive tasks once controlled for IQ. On the other hand, using magnetoencephalography (MEG), Hinkley et al. (2016) observed a reduced language lateralization in AgCC subjects compared to controls during expressive linguistic tasks, as Komaba et al. (1998) reported in one patient studied with positron emission tomography (PET) and a Wada test, and Rieker et al. (2007) in another case studied with fMRI.

Here we explored the role of auditory callosal fibers during the first stages of language acquisition using high-density electroencephalography (EEG, 128 channels) in 3-4-month-old infants with a corpus callosum agenesis compared with typical infants. We studied auditory- 
evoked potentials for speech stimuli presented binaurally (in both ears), monaurally (in one ear) or dichotically (different stimuli in both ears) in $13 \mathrm{AgCC}$ and 18 typically developing infants. We compared the ERP topography but mainly the ERP latencies in both groups. Due to the crossing of the projection pathways in all sensory modalities, the hemisphere contralateral to stimulation is reached first, and information is transferred to the ipsilateral hemisphere via callosal fibers. For the visual modality, the efficiency and maturation of the corpus callosum can be estimated by the delay between contra- and ipsilateral responses since the neural pathways clearly direct each visual hemifield to the contralateral hemisphere (Adibpour, Dubois, \& Dehaene-Lambertz, 2018). However, for the auditory modality, numerous crossings occur at the subcortical level, and the ipsilateral pathway has a strong cortical projection, which makes it difficult to separate the direct ipsilateral response from the response transferred from the contrato the ipsilateral hemisphere through callosal fibers.

We thus reasoned that ERP components depending on the direct ipsilateral and contralateral pathways would have a similar latency in both $\mathrm{AgCC}$ and typical infants, but that any response depending on a callosal transfer would be significantly different between the two groups. Two findings could be considered: 1) because of the transfer delay, ipsilateral responses are slower than contralateral responses in typical infants but not in AgCC infants; 2) ipsilateral latencies are similar in both groups, in which case we may conclude that there is no inter-hemispheric transfer in typical infants at the processing stage we analyzed (P2 component of infant's auditory evoked potentials) due to the immaturity of the corpus callosum. We also studied whether left-to-right and right-to-left transfers were equivalent in $\mathrm{AgCC}$ and typical infants, since in adults, an asymmetric inter-hemispheric transfer of neural information is described between auditory cortices at rest (Andoh, Matsushita, \& Zatorre, 2015) and during auditory motion processing (Krumbholz, Hewson-Stoate, \& Schönwiesner, 2007).

\section{Materials and Methods:}

Subjects:

We studied 13 infants with an agenesis of corpus callosum aged between 10.9 and 18.4 weeks (mean age: $16.3 \pm 2.2 \mathrm{w}, 4$ girls and 9 boys). Agenesis was detected during gestation by ultrasonography monitoring followed by an anatomical MRI to detect other brain anomalies. Three out of the 13 infants had a partial agenesis with at least no splenium, where auditory callosal fibers cross, and the remaining 10 infants had a complete agenesis of the tract. At 2 years of age, developmental quotient was in the normal range (87 to 112), except for one girl who was also dysmorphic and had a dysplasia of the aortic valve. At 8-10 years of age, 9 out of 13 children followed a normal academic curriculum with special help for three of them due to reading difficulties. Therefore, $6 / 13$ had some cognitive difficulties in following a normal school curriculum. By contrast, one of them was particularly gifted at school, being one year ahead in his curriculum. We also studied 18 typical infants (healthy and born full-term) aged between 9.6 and 17 weeks (mean age: $13.9 \pm 2$ w, 8 girls and 10 boys). We had no follow-up for these infants but they should represent the normal population as no difficulties were noted during pregnancy, birth, or the first post-natal months. The study was approved by the regional ethical committee for biomedical research. All parents were informed about its content and goals and gave written informed consent. 


\section{EEG data acquisition:}

EEG was recorded by a 64-electrode-net (EGI, Eugene, USA) referenced to the vertex. The net was placed on the infants' heads relative to anatomical markers, and earphones were placed over the ears to present the auditory stimuli. The infants sat on their parents' laps. To keep them calm, distracting visual stimuli unsynchronized with the auditory stimuli were presented on a screen in front of them. EEG was continuously digitized at a sampling rate of $250 \mathrm{~Hz}$ during the whole experiment (net amp 200 system EGI, Eugene, USA). The experiment was stopped as soon as infants became restless.

\section{Stimuli:}

Two consonant-vowel syllables (/ba/ and /ga/) were produced by a female speaker with the same flat intonation and matched for intensity, total duration $(285 \mathrm{~ms})$, pre-voicing, and voiced formant transition duration (40 and $45 \mathrm{~ms}$ respectively). We further created a 'babble'-like sound (referred to as babbling noise) covering the same range of frequencies, dynamics, and timbre as the syllables by superposing several sentences produced by the same speaker (duration 3s), asynchronously in order to avoid any word recognition.

\section{Experimental paradigm:}

As in our usual design (Dehaene-Lambertz \& Dehaene, 1994), each trial comprised 4 syllables, spaced by an interval of $600 \mathrm{~ms}$. The last syllable was either similar or different from the first three syllables in order to constitute a standard or a deviant condition, and the repeated syllable (/ba/ or /ga/) was randomly chosen for each trial. Syllables were presented either bilaterally or monaurally in the left or right ear. Following a monaural stimulation, both contralateral and ipsilateral pathways contribute to the measured responses, so we aimed to saturate the ipsilateral pathway by simultaneously presenting a complex and continuous stimulation in the opposite ear (Kouider \& Dupoux, 2005). We thus played the babbling noise starting at $600 \mathrm{~ms}$ before the first monaural syllable, which lasted for $3 \mathrm{~s}$, thus during the presentation of the syllable train.

The experiment consisted of 360 trials separated by $2 \mathrm{~s}$ of silence, corresponding to 30 repetitions X 2 conditions ( $4^{\text {th }}$ syllable to be standard or deviant) X 2 syllables (/ba/ $/ \mathrm{ga} /$ ) X 3 sides of presentation (both ears, left ear, right ear). The trial order was randomized. Stimulus presentation and synchronization with the recording system were carried out using the EXPE software (Pallier, Dupoux, \& Jeannin, 1997) on a PC compatible with a Pro-audio Spectrum 16 D/A Board. Syllables were played through earphones at a comfortable hearing level.

\section{EEG pre-processing:}

EEG recordings were band-pass filtered between 0.5 and $20 \mathrm{~Hz}$ using zero-phase lag filter from EEGLAB (Delorme \& Makeig, 2004), and further processed using MATLAB toolboxes: EEGLAB and Brainstorm (Tadel, Baillet, Mosher, Pantazis, \& Leahy, 2011). Two of our initial goals were to compare the ERPs when the syllables were presented bilaterally and monaurally, as well as to study discrimination responses depending on the presented side. However, while the response amplitude is large for the first syllable in this paradigm, it decreases with repetition (Dehaene-Lambertz \& Dehaene, 1994), and here the response to the last syllable was weak, likely attenuated by the superimposed babbling noise. The insufficient signal-to-noise ratio associated with the relatively small number of $\mathrm{AgCC}$ infants prevented robust analyses of the change of syllable. Thus, we focused our comparisons between the AgCC and typical groups on the first syllable of the bilateral and monaural trials, and also on the response to the babbling 
noise. Therefore, the signal was segmented into epochs of $1400 \mathrm{~ms}:[-200,+1200] \mathrm{ms}$ relative to the onset of the first stimulus in the trial (i.e. the first binaural syllable or the babbling noise).

Channels contaminated by movement or eye artifacts were automatically rejected on a trial-bytrial basis based on amplitude variations inside an epoch: each channel epoch was rejected when the fast average amplitude exceeded $250 \mu \mathrm{V}$, or when deviation between fast and slow running averages exceeded $150 \mu \mathrm{V}$. Electrodes were rejected for the entire recording if they were marked as bad in more than $70 \%$ of the epochs, and trials were rejected if more than $50 \%$ of electrodes were marked bad. Recordings were then re-referenced by subtracting the average activity of all channels over the brain to obtain average-reference recordings then baseline-corrected over the first $200 \mathrm{~ms}$ of the segment (i.e. before the onset of babbling noise presentation).

Signals for comparable trials were then averaged together to measure auditory-evoked potentials. In order to increase the signal-to-noise ratio and because we focused on the first syllable, we merged standard and deviant trials, and trials with /ba/ or /ga/ as repeated syllable. It led to 120 trials per side of syllable presentation (left/right/both ear(s)), and after the pre-processing stage, we kept on average 60/60/60 correct trials in the typical group, and 74/74/75 trials in the AgCC group for the left/right/binaural trials.

Analyses of auditory-evoked responses in typical and AgCC infants:

We analyzed the ERPs to three types of sound presentation: 1. binaural stimulation (bilateral presentation of a syllable), 2. monaural babbling noise (focusing on the time period between the noise onset and the presentation of a monaural syllable at $600 \mathrm{~ms}$ ), 3. dichotic stimulation (babbling noise in one ear, a syllable in the other ear, for a time period of $600 \mathrm{~ms}$ after the trial onset).

Comparison of ERPs topographies in typical and AgCC infants: We first aimed to compare the voltage topographies between the two groups. To avoid topography differences being explained by a difference in voltage amplitude rather than a genuine difference over the scalp, we "scaled" the EEG signal in each infant: the signal recorded at each electrode and at each time point was converted to a z-score based on the mean and standard deviation of the time series for all electrodes obtained after averaging all trials over the time-window [-200 1200] ms. For each type of sound presentation (binaural syllables, monaural babbling noise, dichotic stimulation), we performed unpaired t-tests between the two groups on rescaled amplitudes, for each channel, and each time sample during a time-window of $600 \mathrm{~ms}$ from the stimulus onset (either syllables or babbling noise). We first identified clusters showing group differences by clustering neighboring channels and time-samples with a probability below 0.1 and computed their significance probability (noted $\mathrm{p}_{\mathrm{cor}}$ ) using nonparametric statistics (Maris \& Oostenveld, 2007). This was done by performing similar t-test comparisons and cluster extractions for 5000 random permutations of the group labels on the original data and computing pcor based on the number of times the real data produced clusters with higher t-values than the shuffled data. For each of the identified clusters and time windows, we also reported the effect size of amplitude differences between groups using Cohen's d coefficient.

Using the same approach, we also compared the topographical differences between the left and right stimuli within each group. To do so, we compared the response topography to left stimuli with flipped response topography to the right stimuli, i.e. left noise - right noise (flipped) or left syllable - right syllable (flipped). 
Comparison of $P 2$ characteristics in typical and AgCC infants: We secondly compared the two groups based on the $\mathrm{P} 2$ component, since it is the most robust auditory response at this age and it can be identified in each infant contrarily to earlier weaker responses (Wunderlich \& ConeWesson, 2006). This component peaks around 150-350 ms and corresponds to a bilateral positive response over the anterior electrodes synchronous with a bilateral negativity over the posterior electrodes (Dehaene-Lambertz \& Dehaene, 1994). To reliably identify the component in both hemispheres, we considered the best set of electrodes for each group, which differed across groups given the topography differences highlighted by the previous analysis. Based on the topography of the grand average computed over all trials and all infants in each group, these sets were determined to cover the positive pole of the P2 component and consisted of 9 left and right symmetrical electrodes. In typical infants, the two sets comprised 9 electrodes around F3-F7 and F4-F8 in the left and right hemispheres respectively. In AgCC infants, the sets were more lateral, around $\mathrm{T} 7$ and $\mathrm{T} 8$ extending up to $\mathrm{F} 3$ and F4 anteriorly and to P7 and P8 posteriorly.

In each infant and for each type of sound presentation (binaural syllables, monaural babbling noise, dichotic stimulation), P2 was identified as the first distinguishable positive peak on ERPs averaged over the left and right sets independently. We then measured individual P2 latency as well as P2 amplitude from the average amplitude over a $50-\mathrm{ms}$ time window centered on the peak latency of the original, unscaled data.

To compare the typical and AgCC infants, we performed the following analyses of variance (ANOVAs) for each characteristic latency/amplitude as the dependent variable. In the ANOVA for the binaural condition, we considered the group (typical / AgCC) as a between-subject factor, the cerebral hemisphere (left / right) as a within-subject factor, and the interaction between factors. In the ANOVAs for the monaural and dichotic conditions, we considered the brain response side (contralateral/ipsilateral relative to the stimulated ear) as an additional withinsubject factor as well as interactions between the different factors. For significant effects or interactions, we further performed post-hoc analyses using t-tests to detect the significant differences between conditions ( $p$-values were corrected for multiple comparisons using the False Discovery Rate (FDR) approach). We also reported the effect sizes of differences between groups using Cohen's d coefficient.

Comparison of $\mathrm{P} 2$ responses to different stimulations: We finally investigated whether $\mathrm{P} 2$ characteristics were modulated by the type of sound presentation in our paradigm. We thus performed an ANOVA on each characteristic latency/amplitude as the dependent variable with the stimulation type (binaural syllables / monaural babbling noise / dichotic stimulation) and cerebral hemisphere (left/right) as within-subject factors, and group as a between-subject factor (typical / AgCC). For the response to monaural and dichotic stimulations, we averaged the characteristics across the two response sides (contralateral and ipsilateral) in order to keep the same sample size as for the response to binaural stimulation.

\section{Results:}

1) Comparison of ERPs topographies in typical and AgCC infants

We first compared the topographical maps of the left and right stimuli within each group and did not observe any significant difference between the response topographies in any of the infant groups. We then compared the topography of auditory ERPs between typical and AgCC infants using cluster-based analyses for the three types of auditory stimulation (binaural presentation of 
syllables, monaural presentation of babbling noise, dichotic presentation of opposite-side babbling noise and syllables).

As can be seen in figure 1 and also in figure 2.a, the anterior positivity synchronous with a posterior negativity typical of an auditory response at this age was observed in both groups, but the anterior positivity fused at the midline in typical infants. This was not the case in AgCC infants in whom the positivity appeared much more lateral than in controls. These topographical differences were objectivized by the statistical analyses for each type of stimulation.

Binaural stimulation (response to syllables): The difference in topography between both groups was statistically significant over a fronto-medial cluster of 12 electrodes during the time window [396-556] ms post syllable onset $\left(\mathrm{p}_{\mathrm{cor}}=0.031\right.$, Cohen's $\mathrm{d}$ on rescaled amplitudes over the cluster $=1.1$, figure $1 . \mathrm{a}$ ) in which a weaker activity was recorded in AgCC relative to typical infants.

Monaural stimulation (response to babbling noise): As can be seen in figures 1.b and 1.c, the response was more lateral in AgCC than in typically developing infants, yielding a significant difference between groups on a contralateral left temporal cluster when babbling noise was presented in the right ear ( 7 electrodes in the time window [76-568] ms post babbling noise onset, pcor $=0.034$, Cohen's $d=1.4$, figure 1.b). By contrast, no significant difference was observed for babbling noise presented in the left ear, although visual inspection of the ERP shows a similar pattern (figure 1.c).

Dichotic stimulation (response to the syllable in the presence of contralateral babbling noise): Some difference between groups was also observed following dichotic stimulation, but only for one side of presentation, i.e. a trend when the syllable was presented in the left ear (babbling noise in the right ear) over a contralateral right temporal cluster (5 electrodes during the time window [164-404] ms post-dichotic onset, $\mathrm{p}_{\text {cor }}=0.093$, Cohen's $d=1.0$, figure 1.b.), whereas no significant cluster was identified for syllables in the right ear (babbling noise in the left ear) (figure 1.c).

Overall, the difference between the two groups was more visible for the trials in which babbling noise was presented in the right ear and syllables in the left ear. And the comparison of effect sizes suggested that group differences were larger for babbling noise than for syllables.

\section{2) Comparison of P2 latencies in typical and AgCC infants and across brain hemispheres}

The P2 characteristics were reliably measured in each infant over the left and right sets of electrodes, covering the positive peaks in each group (see figure 2 for an example of P2 topography, and supplementary figure 1 for the identification of P2 peaks based on ERP time courses for each type of auditory stimulation). ANOVA results for the P2 latency are summarized below and detailed in table 1 and figure 2 (the complete ANOVA results for the P2 amplitude are presented in Supplementary Information 1 and 2).

Binaural stimulation: We detected no main effect of group (AgCC: $278 \mathrm{~ms}, 95 \%$ CI [259, 297] vs typical: $256 \mathrm{~ms}, 95 \%$ CI [238, 274]), nor hemisphere (left: $264 \mathrm{~ms}$, 95\% CI [242, 286] vs right: $267 \mathrm{~ms}, 95 \%$ CI [251, 283]), nor any interaction between group and hemisphere (figure 2.a; table 1). 
Monaural stimulation (response to babbling noise): We observed a main effect of hemisphere but no main effect of group or response side (see table 1 for complete results). The interactions group $\mathrm{x}$ response side $\mathrm{x}$ hemisphere, group $\mathrm{x}$ hemisphere, and response side $\mathrm{x}$ hemisphere were also significant, while the interaction group $\mathrm{x}$ response side was marginally significant. Post-hoc t-tests of the three-way interactions demonstrated different points. In typical infants, the contralateral response was faster than the ipsilateral response for left ear babbling noise (contralateral: $186 \mathrm{~ms}, 95 \%$ CI [168, 203] vs. ipsilateral: $254 \mathrm{~ms}, 95 \%$ CI [224, 284], p=0.003), whereas for right ear babbling noise, a weak trend suggested faster ipsilateral than the contralateral response (ipsilateral: 169 ms, 95\% CI [148, 190] vs. $202 \mathrm{~ms}$, 95\% CI [178, 225], $\mathrm{p}=0.097)$. The within-hemisphere latency difference for the ipsi- and contralateral babbling noise was significantly larger in the left hemisphere (left: $52 \mathrm{~ms}, 95 \%$ CI [7, 97] vs. right:-16 ms, 95\% CI $[-40,-7], \mathrm{p}=0.045)$ due to the significantly slower ipsilateral response in the left than the right hemisphere (left: $254 \mathrm{~ms}, 95 \%$ CI [224, 284] vs. right $202 \mathrm{~ms}, 95 \%$ CI [178, 225], p=0.002). This slow left ipsilateral response in typical infants was also significantly different from the latency in the same condition in AgCC infants (typical: $254 \mathrm{~ms}, 95 \%$ CI [224, 284] vs. AgCC: $197 \mathrm{~ms}, 95 \%$ CI [177, 218], $\mathrm{p}=0.01)$ suggesting that in typical infants, the left ipsilateral response comprised a transferred component through the corpus callosum but not the right ipsilateral response (figure 2.b). For the $\mathrm{AgCC}$ infants, there was no significant difference between the latencies of the ipsi- and contralateral responses (table 1).

Dichotic stimulation (response to syllables in the presence of contralateral babbling noise): None of the main effects of group, hemisphere, response side, or interactions was significant (figure 2.c; table 1).

To summarize, differences between groups were observed only for a monaural stimulation (i.e. babbling noise), characterized by a slower left ipsilateral response in typical infants relative to AgCC.

\section{3) Different P2 latencies depending on the paradigm of auditory stimulation}

When comparing P2 latencies for the three types of auditory stimulation, we observed a main effect of the stimuli type (respectively 266/201/199 ms, 95\% CI [252, 279] / [192, 210] / [190, 209] for binaural syllables/monaural babbling noise/dichotic syllables) and a main effect of hemisphere (respectively 220/207 ms, 95\% CI [210, 229] / [198, 216] for left/ right stimuli), but no effect of group (see complete results in figure 3 and table 2). We also observed a significant interaction stimuli type $\mathrm{x}$ hemisphere. Post-hoc t-tests analyses revealed a longer latency in both hemispheres for binaural syllables relative to the other two types of stimuli (monaural babbling noise and dichotic babbling noise-syllable trials) whereas the difference between these two latter stimuli was weak (table 2). (The complete ANOVA results for the dependency of the P2 amplitude on the paradigm of auditory stimulation are presented in Supplementary Information 3).

\section{Discussion:}

In this study, we compared infants with corpus callosum agenesis to typical infants in order to understand the role of the callosal fibers in auditory responses during early development, in terms of topography, latency, amplitude, and lateralization. Note that we are studying a late response, the auditory $\mathrm{P} 2$, which is a high-level component originating from associative cortices, mainly because this component can be robustly identified in each individual at this age contrary 
to earlier components (Wunderlich \& Cone-Wesson, 2006). First, we observed a different P2 topography between groups. The anterior positivity extended more toward the lateral temporal regions in AgCC and over the midline in typical infants. Second, we found no difference in amplitudes between ipsi- and contralateral responses nor between typical and AgCC infants, suggesting an efficient and robust ipsilateral auditory pathway at this age. Third, the ipsilateral P2 was slower in typical infants compared to AgCC infants for left-ear but not for right-ear babbling noise. As the ipsilateral response corresponds to both the direct pathway and to the trans-hemispheric transfer, this difference in responses to a lateralized sound indicates an asymmetric transfer of the auditory responses between hemispheres. Our AgCC group included three children with partial agenesis. Our rational was that it should not affect our analyses given that auditory callosal fibers were inexistent in these infants since they cross at the level of the missing part of the corpus callosum, i.e. its posterior portion. Nevertheless, analyses conducted without these infants provided similar results (see Supplementary Information 4), although with a drop in the significance due to the reduced number of subjects.

\section{Altered topography of auditory responses in AgCC infants}

The corpus callosum is a large tract connecting both hemispheres. In the case of agenesis, the would-be callosal fibers, which no longer cross the midline, become oriented along the anteriorposterior direction, constituting an aberrant tract, the Probst fibers. A recent tractography study in 12 children with corpus callosum dysgenesis revealed the complex connectivity of these fibers, which is not limited to frontal-occipital regions (Bénézit et al., 2015). Numerous fibers along the Probst main tract connect temporal and parietal areas. The fact that the Probst fibers do not degenerate and furthermore have similar DTI characteristics as remnants of the corpus callosum in the case of partial callosal agenesis (Bénézit et al., 2015) prove that they are functional and may propagate activity within the hemisphere to unexpected areas altering the voltage topography on the scalp.

Additionally, the absence of crossing callosal fibers affects the gyration of the cortex, the most obvious difference from a typical brain gyration being the orientation of the sulci on the medial surface of the brain which is vertical instead of wrapped around the corpus callosum. At a microstructural level, the lack of callosal fibers might also change the vector orientation of the electric field within the cortical columns. All these factors might affect the surface topography in AgCC subjects. Unfortunately, without an MRI in each infant, a correct reconstruction of the active sources of the auditory P2 was not possible. However, the group difference for the frontal positivity is congruent with the proposal of a source in the anterior cingulate cortex in complement of those in the superior temporal cortices at the origin of the P2 (Ortiz-Mantilla, Hämäläinen, \& Benasich, 2012). Indeed, it is at this level that the difference of gyration is maximal between groups and thus might affect the surface topography most strongly.

\section{A strong contribution of the ipsilateral pathway to auditory processing in the developing brain}

The input to each ear travels to both hemispheres through ipsi- and contralateral pathways. The responses are subsequently transferred from one hemisphere to the other through the callosal fibers. Subjects lacking callosal splenial fibers are thus the only adequate model to evaluate the respective contribution of contra- and ipsilateral pathways on auditory ERPs. In human adults and animals, contralateral pathways are generally predominant relative to ipsilateral pathways (Majkowski et al., 1971; Rosenzweig, 1951) until the auditory cortices; however, depending on 
the task (e.g. attention oriented toward on ear) and on the stimulus (e.g. speech vs music), the predominance of one hemisphere over the other might vary at the cortical level. Here, in AgCC infants, the P2 following monaural babbling noise was simultaneously recorded over both hemispheres, revealing that both pathways were equally efficient at three months of age. The lack of differences in amplitude on the clusters ipsi- and contralateral to the stimulated ears further emphasizes the strength of the ipsilateral response at this age. As there was also no difference in ERP amplitude between AgCC and typical infants (see Supplementary Information 1), we may hypothesize that it is the common rule at this age. Our stimulus presentation was passive, and all stimuli were speech, limiting the generalization of our results, yet we show here that a robust ipsilateral pathway ensures that both hemispheres are a-priori similarly reachable by the auditory stimulation without inter-hemispheric connectivity. It may explain why linguistic left-lateralization, especially in receptive tasks, is similar in AgCC adults and in controls (Pelletier et al., 2011).

\section{Asymmetry of inter-hemispheric connections}

To grasp the role of the corpus callosum, we compared AgCC and typical infants listening to monaural babbling noise. The two groups notably differed in the latency of the ipsilateral left response when babbling noise was presented in the left ear. We interpreted the delay in the peak of the ipsilateral left P2 as resulting from a superposition of the ipsilateral direct response and the inter-hemispheric transfer of the contralateral response. Taking either the lag between the contraand ipsilateral responses in typical infants $(69 \mathrm{~ms})$ or the difference between AgCC and typical infants $(57 \mathrm{~ms})$ for the ipsilateral left response provides a similar estimation of the interhemispheric transfer time of auditory information at this age. Although slow relative to adult IHTT values, which are estimated to be a few ms (from $3 \mathrm{~ms}$ for tactile stimuli (Tame \& Longo., 2015) to 10-30 ms for visual stimuli (Saron \& Davidson, 1989; Whitford et al., 2011) depending on their complexity), a value of $\sim 60 \mathrm{~ms}$ is in agreement with the values we obtained for the visual P1 transfer around the same age, the distance being longer for a visual transfer than for an auditory transfer. Using visual hemifield presentation of faces, we measured an acceleration of the IHTT during the first post-natal semester from around $315 \mathrm{~ms}$ at six weeks to $84 \mathrm{~ms}$ four months later (Adibpour, Dubois and Dehaene-Lambertz. 2018). By contrast, it was surprising to record no difference between groups for right-side babbling noise, suggesting that there was little left-to-right inter-hemispheric transfer at this age for this type of auditory information. In the experiment cited above (Adibpour, Dubois and Dehaene-Lambertz. 2018), there was no asymmetry in the transfer time for the visual P1. However in adults, a similar asymmetry in the auditory inter-hemispheric connectivity was described: TMS applied over the right but not the left auditory cortex changed the functional connectivity between auditory cortices during restingstate in proportion to the volume of auditory callosal fibers (Andoh et al., 2015). Similarly, Gotts et al (2013) reported stronger functional connectivity within the left hemisphere, especially for regions located within the language network, whereas the right hemisphere interacts more widely with both hemispheres (Gotts et al., 2013). This asymmetrical pattern may therefore find its roots during early development.

How this asymmetry in inter-hemispheric communications affects language lateralization is not yet clear. It might facilitate language learning by integrating all information in the same areas instead of distributing resources in both hemispheres. Although brain plasticity is sufficient to allow linguistic reorganization in the right hemisphere in the case of an early left-sided lesion (Bates et al, 1999), competing hemispheres might be deleterious when attentional resources are 
limited as is the case in young children. It may explain why AgCC children may have learning difficulties as pointed out by Sander (1989) and Temple et al $(1989,1993)$ and as seen in our cohort in which 6 out of 13 required extra help to follow their primary school cursus. However, corpus callosum agenesis might also be the visible symptom of more diffuse neural anomalies not seen with currently available MRI, which alleviates the hypothesis of a unique role of corpus callosum transfer in these difficulties.

We might have expected a similar effect for the subsequent syllable presented in the other ear than the babbling noise, but there was no difference between groups nor delays of the ipsilateral left response for a left-ear syllable. However, in this case, the left hemisphere was saturated by the continuous babbling noise in the right ear projecting to the left hemisphere and blocking the right-to-left transfer. Still, no evidence of a left-to-right transfer was observed. Although the amplitude was much weaker for the dichotic syllables relative to the same syllables presented binaurally likely due to the masking babbling noise, the P2 latency was shorter ( 66 $\mathrm{ms})$. We interpret this acceleration of the P2 peak as the attention trigger that preceded the babbling noise in $2 / 3$ of the trials.

\section{Conclusions:}

Our study on the developing auditory network reveals an asymmetry between left-to-right and right-to-left effective connections, whereas the current structural approaches (e.g. diffusion imaging combined with tractography) cannot dissociate them. Our conclusions rely on the simple hypothesis that infants with corpus callosum agenesis have a similar auditory network except the missing corpus callosum. While our conclusions may be oversimplified, our interpretation is sensible, given the relative similarity in latencies and amplitudes of the auditory responses between both groups aside the notable exception of the left ipsilateral response for monaural stimulation we have discussed above. This result enters in the now large amount of evidence showing that the human brain architecture is lateralized very early on and differently depending on the cognitive domain and brain areas. Why evolution has selected this asymmetric architecture and how it contributes to the development of complex cognitive functions remains to be understood.

Acknowledgements: The authors would like to thank all the infants and their parents who participated in this study. We are also grateful to Claire Kabdebon for her help in EEG analyses and Eric Moulton for proofreading the text.

Funding: This research was supported by grants from the Fondation de France (to J. D and G. DL), NRJ-Institut de France (to G. D-L) and European Research Council (BabyLearn grant to G. D-L).

Conflict of Interest: The authors declare that they have no conflict of interest.

Ethical approval: All procedures performed in studies involving human participants were in accordance with the ethical standards of the institutional and/or national research committee and with the 1964 Helsinki declaration and its later amendments or comparable ethical standards.

Informed consent: Written informed consent was obtained from all infants' parents included in the study. 


\section{References:}

Aboitiz, F., Scheibel, A. B., Fisher, R. S., \& Zaidel, E. (1992). Fiber composition of the human corpus callosum. Brain research, 598(1), 143-153.

Adibpour, P., Dubois, J., \& Dehaene-Lambertz, G. (2018). Right but not left hemispheric discrimination of faces in infancy. Nature Human Behavior 2 67-79.

Andoh, J., Matsushita, R., \& Zatorre, R. J. (2015). Asymmetric interhemispheric transfer in the auditory network: evidence from TMS, resting-state fMRI, and diffusion imaging. Journal of neuroscience, 35(43), 14602-14611.

Bates, E., Vicari, S., \& Trauner, D. (1999). Neural mediation of language development: Perspectives from lesion studies of infants and children.

Bedeschi, M. F., Bonaglia, M. C., Grasso, R., Pellegri, A., Garghentino, R. R., Battaglia, M. A., . . . Bresolin, N. (2006). Agenesis of the corpus callosum: clinical and genetic study in 63 young patients. Pediatric neurology, 34(3), 186-193.

Bénézit, A., Hertz-Pannier, L., Dehaene-Lambertz, G., Monzalvo, K., Germanaud, D., Duclap, D., . . Moutard, M.-L. (2015). Organising white matter in a brain without corpus callosum fibres. Cortex, 63, 155-171.

Bonneau, D., Toutain, A., Laquerriere, A., Marret, S., Saugier-Veber, P., Barthez, M. A., . . . Gélot, A. (2002). X-linked lissencephaly with absent corpus callosum and ambiguous genitalia (XLAG): clinical, magnetic resonance imaging, and neuropathological findings. Annals of neurology, 51(3), 340-349.

Brody, B. A., Kinney, H. C., Kloman, A. S., \& Gilles, F. H. (1987). Sequence of central nervous system myelination in human infancy. I. An autopsy study of myelination. Journal of Neuropathology \& Experimental Neurology, 46(3), 283-301.

Caminiti, R., Ghaziri, H., Galuske, R., Hof, P. R., \& Innocenti, G. M. (2009). Evolution amplified processing with temporally dispersed slow neuronal connectivity in primates. Proc Natl Acad Sci U S A, 106(46), 19551-19556. doi:10.1073/pnas.0907655106

Chiarello, C. (1980). A house divided? Cognitive functioning with callosal agenesis. Brain and Language, 11(1), 128-158.

Cook, N. D. (1984). Homotopic callosal inhibition. Brain and Language, 23(1), 116-125.

de Schonen, \& Mathivet, E. (1990). Hemispheric asymmetry in a face discrimination task in infants. Child Dev, 61(4), 1192-1205.

Dehaene-Lambertz, G., \& Dehaene, S. (1994). Speed and cerebral correlates of syllable discrimination in infants. Nature, 370(6487), 292.

Dehaene-Lambertz, G., Dehaene, S., \& Hertz-Pannier, L. (2002). Functional neuroimaging of speech perception in infants. Science, 298(5600), 2013-2015. 
Dehaene-Lambertz, G., Pena, M., Christophe, A., \& Landrieu, P. (2004). Phoneme perception in a neonate with a left sylvian infarct. Brain Lang, 88(1), 26-38.

Delorme, A., \& Makeig, S. (2004). EEGLAB: an open source toolbox for analysis of single-trial EEG dynamics including independent component analysis. Journal of neuroscience methods, 134(1), 9-21.

Dennis, M. (1981). Language in a congenitally acallosal brain. Brain and Language, 12(1), 33-53.

Dubois, Hertz-Pannier, L., Cachia, A., Mangin, J., Le Bihan, D., \& Dehaene-Lambertz, G. (2009). Structural asymmetries in the infant language and sensori-motor networks. Cerebral cortex, $19(2), 414-423$.

Dubois, J., Benders, M., Lazeyras, F., Borradori-Tolsa, C., Leuchter, R. H.-V., Mangin, J.-F., \& Hüppi, P. S. (2010). Structural asymmetries of perisylvian regions in the preterm newborn. Neuroimage, 52(1), 32-42.

Dubois, J., Poupon, C., Thirion, B., Simonnet, H., Kulikova, S., Leroy, F., . . . Dehaene-Lambertz, G. (2016). Exploring the early organization and maturation of linguistic pathways in the human infant brain. Cerebral cortex, 26(5), 2283-2298.

Evrard, S. G., Vega, M. D., Ramos, A. J., Tagliaferro, P., \& Brusco, A. (2003). Altered neuron-glia interactions in a low, chronic prenatal ethanol exposure. Developmental brain research, 147(1), 119-133.

Glasel, H., Leroy, F., Dubois, J., Hertz-Pannier, L., Mangin, J.-F., \& Dehaene-Lambertz, G. (2011). A robust cerebral asymmetry in the infant brain: the rightward superior temporal sulcus. Neuroimage, 58(3), 716-723.

Gotts, S. J., Jo, H. J., Wallace, G. L., Saad, Z. S., Cox, R. W., \& Martin, A. (2013). Two distinct forms of functional lateralization in the human brain. Proc Natl Acad Sci U S A, 110(36), E34353444. doi:10.1073/pnas.1302581110

Guillem, P., Fabre, B., Cans, C., Robert-Gnansia, E., \& Jouk, P. (2003). Trends in elective terminations of pregnancy between 1989 and 2000 in a French county (the Isere). Prenatal diagnosis, 23(11), 877-883.

Habas, P. A., Scott, J. A., Roosta, A., Rajagopalan, V., Kim, K., Rousseau, F., . . Studholme, C. (2012). Early folding patterns and asymmetries of the normal human brain detected from in utero MRI. Cereb Cortex, 22(1), 13-25. doi:10.1093/cercor/bhr053

Innocenti, G. M. (1986). General organization of callosal connections in the cerebral cortex Sensory-motor areas and aspects of cortical connectivity (pp. 291-353): Springer.

Jeeves, M., \& Temple, C. (1987). A further study of language function in callosal agenesis. Brain and Language, 32(2), 325-335.

Karbe, H., Herholz, K., Halber, M., \& Heiss, W. D. (1998). Collateral inhibition of transcallosal activity facilitates functional brain asymmetry. J Cereb Blood Flow Metab, 18(10), 1157-1161. doi:10.1097/00004647-199810000-00012 
Kostović, I., \& Jovanov-Milošević, N. (2006). The development of cerebral connections during the first 20-45 weeks' gestation. Paper presented at the Seminars in Fetal and Neonatal Medicine.

Kouider, S., \& Dupoux, E. (2005). Subliminal speech priming. Psychological Science, 16(8), 617625.

Krumbholz, K., Hewson-Stoate, N., \& Schönwiesner, M. (2007). Cortical response to auditory motion suggests an asymmetry in the reliance on inter-hemispheric connections between the left and right auditory cortices. Journal of neurophysiology, 97(2), 1649-1655.

Leroy, F., Glasel, H., Dubois, J., Hertz-Pannier, L., Thirion, B., Mangin, J.-F., \& Dehaene-Lambertz, G. (2011). Early maturation of the linguistic dorsal pathway in human infants. Journal of neuroscience, 31(4), 1500-1506.

Liégeois, F., Bentejac, L., \& de Schonen, S. (2000). When does inter-hemispheric integration of visual events emerge in infancy? A developmental study on 19-to 28-month-old infants. Neuropsychologia, 38(10), 1382-1389.

Mahmoudzadeh, M., Dehaene-Lambertz, G., Fournier, M., Kongolo, G., Goudjil, S., Dubois, J., . . - Wallois, F. (2013). Syllabic discrimination in premature human infants prior to complete formation of cortical layers. Proceedings of the National Academy of Sciences, 110(12), 48464851.

Majkowski, J., Bochenek, Z., Bochenek, W., Knapik-Fijałkowska, D., \& Kopeć, J. (1971). Latency of averaged evoked potentials to contralateral and ipsilateral auditory stimulation in normal subjects. Brain research, 25(2), 416-419.

Maris, E., \& Oostenveld, R. (2007). Nonparametric statistical testing of EEG-and MEG-data. Journal of neuroscience methods, 164(1), 177-190.

Ortiz-Mantilla, S., Hämäläinen, J. A., \& Benasich, A. A. (2012). Time course of ERP generators to syllables in infants: a source localization study using age-appropriate brain templates. Neuroimage, 59(4), 3275-3287.

Pallier, C., Dupoux, E., \& Jeannin, X. (1997). EXPE: An expandable programming language for online psychological experiments. Behavior Research Methods, 29(3), 322-327.

Paul, L. K., Brown, W. S., Adolphs, R., Tyszka, J. M., Richards, L. J., Mukherjee, P., \& Sherr, E. H. (2007). Agenesis of the corpus callosum: genetic, developmental and functional aspects of connectivity. Nature Reviews Neuroscience, 8(4), 287-299.

Pelletier, I., Paquette, N., Lepore, F., Rouleau, I., Sauerwein, C. H., Rosa, C., . . . Lassonde, M. (2011). Language lateralization in individuals with callosal agenesis: an fMRI study. Neuropsychologia, 49(7), 1987-1995. doi:10.1016/j.neuropsychologia.2011.03.028

Pena, M., Maki, A., Kovačić, D., Dehaene-Lambertz, G., Koizumi, H., Bouquet, F., \& Mehler, J. (2003). Sounds and silence: an optical topography study of language recognition at birth. Proceedings of the National Academy of Sciences, 100(20), 11702-11705. 
Ringo, J. L., Doty, R. W., Demeter, S., \& Simard, P. Y. (1994). Time is of the essence: a conjecture that hemispheric specialization arises from interhemispheric conduction delay. Cereb Cortex, $4(4), 331-343$.

Rosenzweig, M. R. (1951). Representations of the two ears at the auditory cortex. American Journal of Physiology--Legacy Content, 167(1), 147-158.

Sanders, R. J. (1989). Sentence comprehension following agenesis of the corpus callosum. Brain and Language, 37(1), 59-72.

Saron, C. D., \& Davidson, R. J. (1989). Visual evoked potential measures of interhemispheric transfer time in humans. Behavioral neuroscience, 103(5), 1115.

Selnes, O. A. (1974). The corpus callosum: Some anatomical and functional considerations with special reference to language. Brain and Language, 1(2), 111-139.

Shultz, S., Vouloumanos, A., Bennett, R. H., \& Pelphrey, K. (2014). Neural specialization for speech in the first months of life. Developmental Science, 17(5), 766-774.

Tadel, F., Baillet, S., Mosher, J. C., Pantazis, D., \& Leahy, R. M. (2011). Brainstorm: a userfriendly application for MEG/EEG analysis. Computational intelligence and neuroscience, 2011, 8.

Tamè, L., \& Longo, M. R. (2015). Inter-hemispheric integration of tactile-motor responses across body parts. Frontiers in human neuroscience, 9, 345.

Temple, C., \& Ilsleya, J. (1993). Phonemic discrimination in callosal agenesis. Cortex, 29(2), 341348.

Temple, C., Jeeves, M., \& Vilarroya, O. (1989). Ten pen men: Rhyming skills in two children with callosal agenesis. Brain and Language, 37(4), 548-564.

Toga, A. W., \& Thompson, P. M. (2003). Mapping brain asymmetry. Nature Reviews Neuroscience, 4(1), 37-48.

Van Essen, D. C. (2005). A population-average, landmark-and surface-based (PALS) atlas of human cerebral cortex. Neuroimage, 28(3), 635-662.

Wahl, M., Strominger, Z., Jeremy, R. J., Barkovich, A. J., Wakahiro, M., Sherr, E. H., \& Mukherjee, P. (2009). Variability of homotopic and heterotopic callosal connectivity in partial agenesis of the corpus callosum: a $3 \mathrm{~T}$ diffusion tensor imaging and Q-ball tractography study. American Journal of Neuroradiology, 30(2), 282-289.

Whitford, T. J., Kubicki, M., Ghorashi, S., Schneiderman, J. S., Hawley, K. J., McCarley, R. W., . . . Spencer, K. M. (2011). Predicting inter-hemispheric transfer time from the diffusion properties of the corpus callosum in healthy individuals and schizophrenia patients: a combined ERP and DTI study. Neuroimage, 54(3), 2318-2329.

Wunderlich, J. L., \& Cone-Wesson, B. K. (2006). Maturation of CAEP in infants and children: a review. Hearing research, 212(1), 212-223. 
Yakovlev., \& Lecours. (1967). The myelogenetic cycles of regional maturation in the brain. : Oxford: Blackwell.

Yazgan, M. Y., Wexler, B. E., Kinsbourne, M., Peterson, B., \& Leckman, J. F. (1995). Functional significance of individual variations in callosal area. Neuropsychologia, 33(6), 769-779.

\section{Figure 1: Topographical differences between typical and AgCC infants:}

ERP time courses of the grand average responses in typical (black) and AgCC (magenta) infants for a) binaural trials; b) dichotic trials: right-ear babbling noise / left-ear syllable trials; c) dichotic trials: left-ear babbling noise / right-ear syllable. In a) and b), significant differences between both groups are observed for the highlighted clusters and over the time-windows shaded in light blue (asterisk correspond to $\mathrm{p}<0.05$, m.s: marginally significant trends $\mathrm{p}<0.1$ ). In $\mathrm{c}$ ), no group difference was observed, thus we highlighted symmetrical outlined clusters compared to b) (n.s.: non-significant). 2D voltage topographies correspond to the shaded time-windows filled with diagonal stripes.

\section{Binaural Syllables}

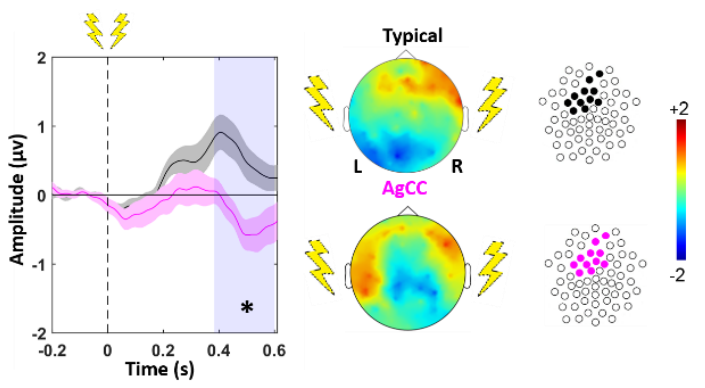

b.

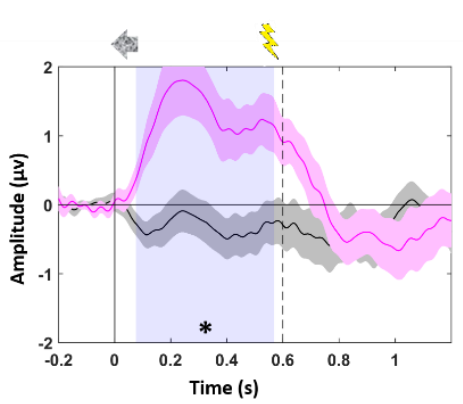

Right Noise - Left Syllable
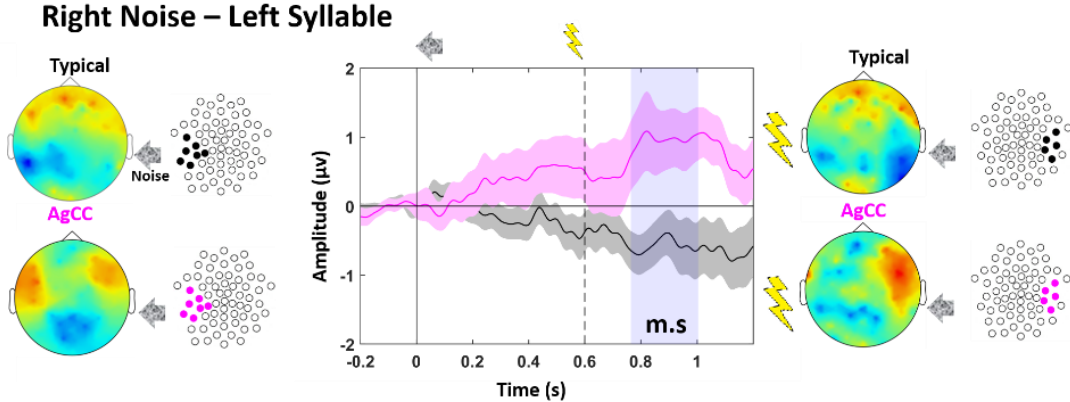

c.

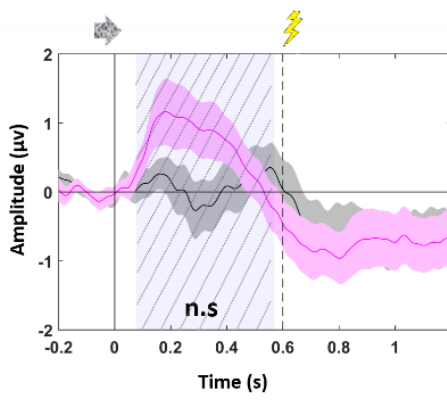

Left Noise - Right Syllable
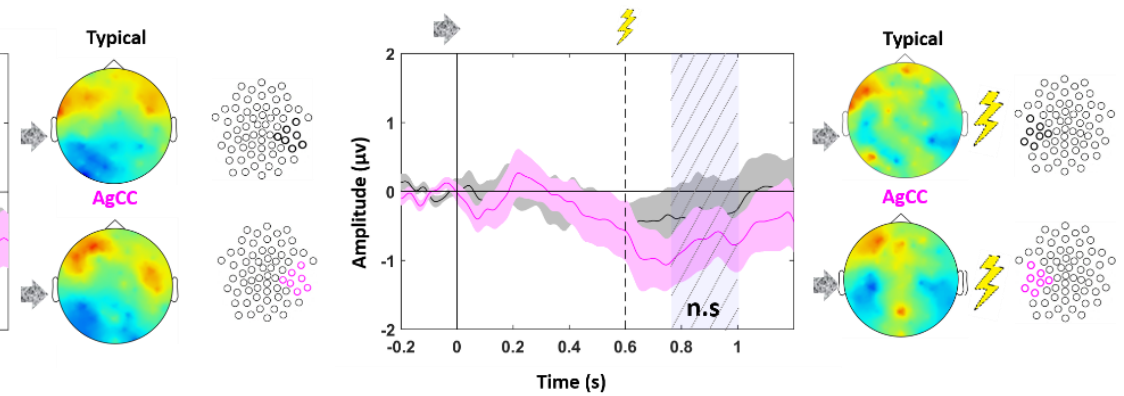
Figure 2: Comparison of P2 response latency between typical and AgCC infants:

a) Grand average ERP time courses in response to monaural babbling noise presented to the left ear, averaged over left (red) and right (blue) sets of electrodes optimized for typical (left panel) and $\mathrm{AgCC}$ (right panel) infants (averaged ERP time courses for other stimuli are shown in supplementary figure 1). P2 latency was measured as the delay between the stimulus onset and the first positive peak. Box plots represent the latency of P2 responses measured in typical (black) and $\mathrm{AgCC}$ (magenta) infants, in each hemisphere, following stimulation by b) binaural syllables, c) monaural babbling noise and d) dichotic babbling noise-syllable. Bilateral responses are considered in $b$ ), while both contralateral and ipsilateral responses are shown in c) and d). Differences between response latencies are highlighted with asterisks ( $\left.{ }^{*} \mathrm{p}<0.05 ; * * \mathrm{p}<0.005\right)$.

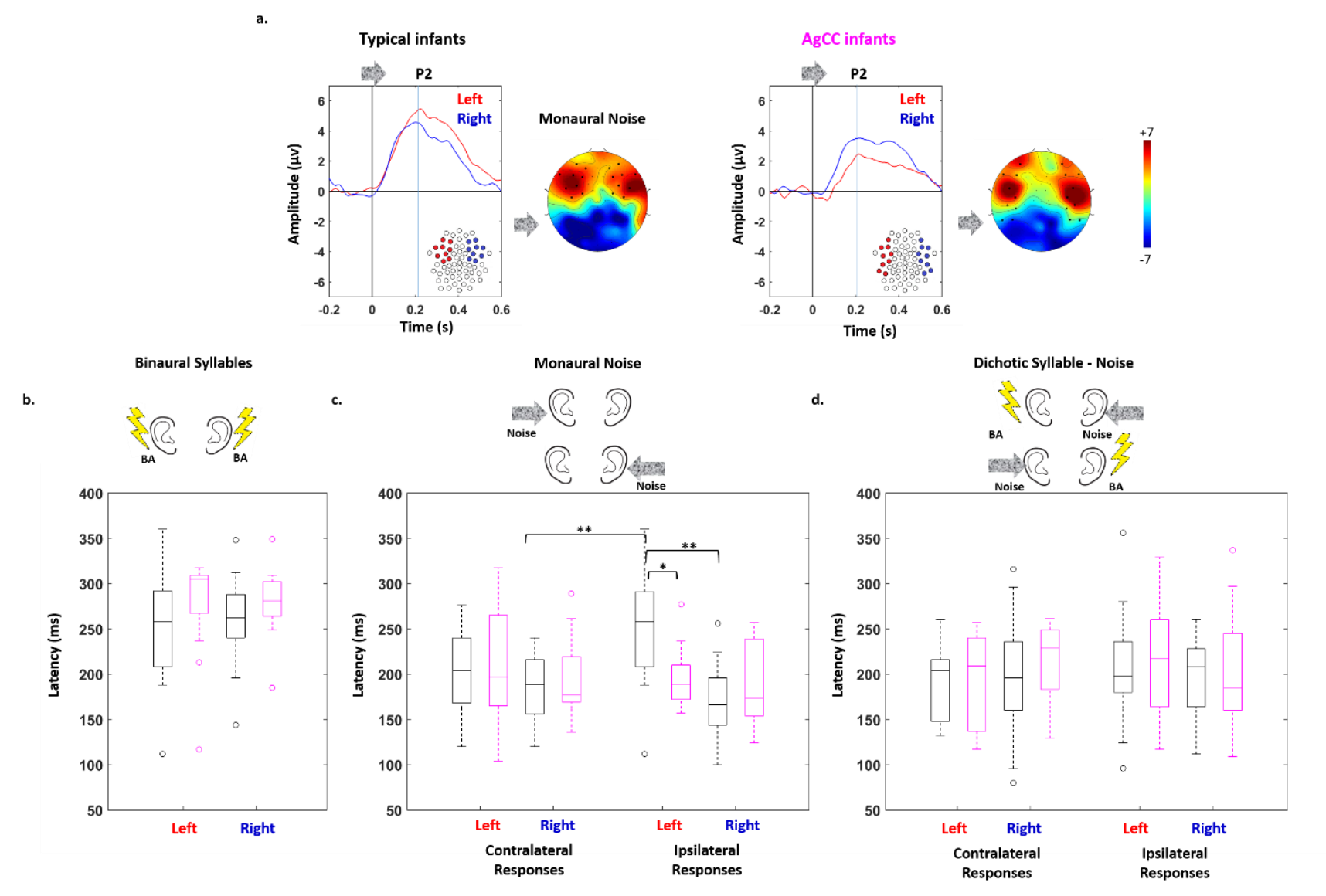


Figure 3: Influence of the type of stimulation on P2 response latency:

Box plots represent the P2 latencies averaged over both hemispheres (left and right sets of electrodes, corresponding to both contralateral and ipsilateral responses) in response to binaural syllables, monaural noise and dichotic babbling noise-syllables in typical (black) and AgCC (magenta) infants. Differences between response latencies are highlighted with asterisks $(* *$ $\mathrm{p}<0.005 ; * \mathrm{p}<0.5)$, showing that responses for binaural syllables are slower than for other stimuli.

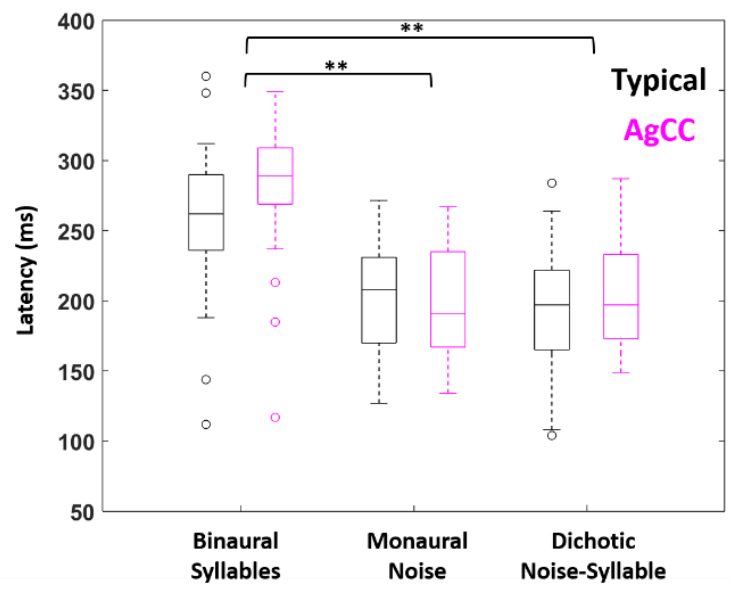

Table 1: ANOVAs of P2 response latency in the binaural, monaural and dichotic trials:

For each ANOVA, the main effects and their interactions are first reported, followed by post-hoc analyses using t-tests when interactions were significant. Effect sizes (Cohen's d) and 95\% confidence intervals (CI) on the averaged differences are indicated. Significant p-values, corrected for multiple comparisons are highlighted by asterisks: $* * \mathrm{p}<0.005$; $* \mathrm{p}<0.5$. AgCC: agenesis of corpus callosum, ipsi: ipsilateral, contra: contralateral, L: left hemisphere, R: right hemisphere.

\begin{tabular}{|c|c|c|}
\hline Binaural & Monaural & Dichotic \\
\hline group : $F(1,29)=1.8, p=0.191$ & group : $F(1,29)=0.1, p=0.703$ & group : $F(1,29)=0.5, p=0.481$ \\
\hline \multirow[t]{2}{*}{ hemisphere: $F(1,29)=0.1, p=0.719$} & hemisphere : $F(1,29)=21.9, p<0.001^{* *}$ & hemisphere: $F(1,29)<0.1, p=0.993$ \\
\hline & response side : $F(1,29)=0.6, p=0.459$ & response side : $F(1,29)=0.6, p=0.424$ \\
\hline \multirow[t]{5}{*}{ group $x$ hemisphere : $F(1,29)<0.1, p=0.86$} & group $x$ hemisphere : $F(1,29)=5.7, p=0.023 *$ & group $\times$ hemisphere : $F(1,29)=0.2, p=0.626$ \\
\hline & group $\times$ response side $: F(1,29)=3.7, p=0.065$ & group $\times$ response side $: F(1,29)<0.1, p=0.928$ \\
\hline & hemisphere $x$ response side : $F(1,29)=4.4, p=0.045^{*}$ & hemisphere $x$ response side $: F(1,29)=1.7, p=0.200$ \\
\hline & group $\times$ hemisphere $\times$ response side : $F(1,29)=5.3, p=0.029 *$ & group $x$ hemisphere $x$ response side $: F(1,29)=0.2, p=0.689$ \\
\hline & 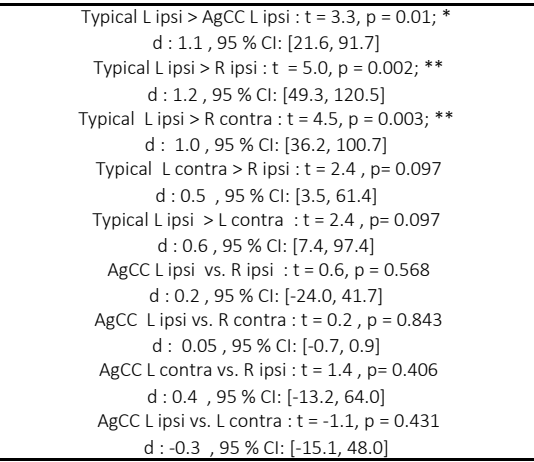 & \\
\hline
\end{tabular}


Table 2: Summary of ANOVA analyses on P2 latencies in function of the type of auditory stimulation:

The main effects and their interactions are first reported, followed by post-hoc analyses using ttests. Effect sizes (Cohen's d) and 95\% confidence interval (CI) on averaged differences are indicated. Significant p-values, corrected for multiple comparisons are highlighted by asterisks: $* * \mathrm{p}<0.005 ; * \mathrm{p}<0.5$. bin:binaural; mon:monaural

\begin{tabular}{|c|}
\hline Dependency of P2 latencies on auditory stimulation \\
\hline stimuli type : $F(1,58)=50.1, p<0.001 * *$ \\
\hline group : $F(1,29)=0.8, p=0.385$ \\
\hline hemisphere : $F(1,58)=4.5, p=0.042 *$ \\
\hline stimuli type $x$ group : $F(2,58)=1.4, p=0.255$ \\
\hline stimuli type $x$ hemisphere $: F(2,58)=7.1, p=0.002 * *$ \\
\hline group $x$ hemisphere $: F(2,58)=1.8, p=0.190$ \\
\hline stimuli type $x$ group $x$ hemisphere $: F(2,58)=1.6, p=0.210$ \\
\hline $\begin{array}{r}\text { bin syllable left }>\text { mon babbling left: } \mathrm{t}(1,30)=4.5, \mathrm{p}<0.001^{* *} ; \\
\text { d: } 0.8,95 \% \mathrm{Cl}:[24.9,65.8] \\
\text { bin syllable right }>\text { mon babbling right: } \mathrm{t}(1,30)=9.4, \mathrm{p}<0.001^{* *} ; \\
\text { d: } 1.7,95 \% \mathrm{Cl}:[65.7,102.3] \\
\text { bin syllable left }>\text { dichotic syllable left: } \mathrm{t}(1,30)=5.8, \mathrm{p}<0.001^{* *} ; \\
\mathrm{d}: 1.0,95 \% \mathrm{Cl}:[41.6,86.9] \\
\text { bin syllable right }>\text { dichotic syllable right: } \mathrm{t}(1,30)=8.0, \mathrm{p}<0.001^{* *} ; \\
\text { d: } 1.4,95 \% \mathrm{Cl}:[50.5,84.9] \\
\text { mon babbling left vs. dichotic syllable left: } \mathrm{t}(1,30)=2.1, \mathrm{p}=0.059 ; \\
\text { d: } 0.4,95 \% \mathrm{Cl}:[0.4,37.4] \\
\text { mon babbling right vs. dichotic syllable right: } \mathrm{t}(1,30)=-1.7, \mathrm{p}=0.088 ; \\
\text { d: } 0.3,95 \% \mathrm{Cl}:[-35.2,2.6]\end{array}$ \\
\hline
\end{tabular}




\section{Supplementary Information:}

We performed ANOVAs on the P2 amplitudes, using the same factors as the ANOVAs performed on P2 latencies.

1. Do P2 amplitudes differ in typical and AgCC infants apart from differences in ERPs topographies?

We compared both groups for potential differences in their P2 response amplitude in response to the different stimuli (binaural, monaural and dichotic stimuli). Supplementary figure 1 illustrates the time course of the grand average ERPs for both groups of infants, providing a schematic view of the P2 time-course. No significant difference between the two groups was revealed by the ANOVAs. Supplementary figure 2 and supplementary table 1 summarize the statistical analyses.

\section{Are P2 amplitudes in response to monaural stimuli stronger over the contralateral compared to the ipsilateral hemisphere?}

Finally, because the ANOVA revealed a significant difference in voltage topography between groups only for right-ear babbling noise, we investigated the amplitude of the ipsi- and contralateral responses to left and right babbling noise in both groups with paired t-tests. In typical infants, the P2 amplitude was similar over the left and right hemispheres for the contralateral $(\mathrm{t}(1,17)=-1.3, \mathrm{p}=0.194$, Cohen's $\mathrm{d}$ : $-0.3,95 \% \mathrm{CI}$ : [-4.8 1.0]) and ipsilateral response $(\mathrm{t}(1,17)=1.3, \mathrm{p}=0.192$, Cohen's d: 0.3, $95 \% \mathrm{CI}$ : [-1.1 5.0]). In AgCC infants, the P2 amplitude was also similar over the left and right hemispheres for both the contralateral $(\mathrm{t}(1,12)$ $=0.3, \mathrm{p}=0.750$, Cohen's $\mathrm{d}=0.1,95 \% \mathrm{CI}$ : [-2.4 3.2]) and ipsilateral responses $(\mathrm{t}(1,12)=0.6, \mathrm{p}$ $=0.551$, Cohen's $\mathrm{d}=0.2,95 \% \mathrm{CI}:[-1.42 .5])$.

\section{Do P2 amplitudes depend on auditory stimulation?}

ANOVAs on the P2 amplitude with the type of auditory stimulation revealed a main effect of stimuli type but no effect of group or hemisphere. No interaction was significant. Post-hoc analyses demonstrated that the P2 amplitude was significantly weaker in response to dichotic stimulation compared to binaural stimulation and monaural stimulation, likely due to the masking effect of the babbling noise in the other ear. The P2 amplitude was also weaker in response to binaural syllables relative to monaural babbling noise stimuli, but both stimuli were highly different in complexity, length, and intensity envelope. These results are illustrated in supplementary figure 3 and supplementary table 2 .

4. Are the responses in AgCC infant group affected by the infants with partial agenesis of callosal fibers?

The AgCC infant group included 3 infants with partial agenesis of callosal fibers. In our study, it seemed rational to include those infants when we focused on auditory perception due to the following reasons: 1 . The auditory callosal fibers cross the brain midline in the posterior portion of the corpus callosum which was missing in all our infants, even the ones with partial ACC. 2. We verified that our group differences were not greatly affected when we removed these 3 infants from the group. Note that removing 3 on 13 infants (almost $25 \%$ ) should clearly affect the sensitivity of the statistical analyses.

Examining the topographical differences between typical and complete AgCC infants (excluding the partial AgCC infants), revealed that despite the drop in the significance of the results, the 
overall trend was preserved. Non-parametric cluster analyses identified smaller clusters of electrodes during shorter time-windows, i.e. [440-592] $\mathrm{ms}$ for binaural syllables $(\mathrm{p}=0.087)$, [152-320] ms for right monaural noise $(p=0.192)$ and [144 344] ms for left dichotic syllables $(p$ $=0.0978)$. No significant cluster was found for left monaural noise and right dichotic syllables (supplementary figure 4).

Regarding the analyses of P2 latency and amplitude, we observed similar results as before without the 3 latter infants (statistical analyses are presented in supplementary tables 3 and 4).

\section{Supplementary figure 1: Auditory-evoked responses to different stimuli in typical and AgCC infants:}

ERP time course of the grand averages recorded in typical (left panel) and AgCC infants (right panel), corresponding to: a) binaural trials; b) trials with syllables in the left ear and babbling noise in the right ear; c) trials with syllables in the right ear and babbling noise in the left ear. The voltage was averaged across the channels highlighted in red (left cluster) and blue (right cluster) on the channel maps presented on the top row. These clusters were located on the P2 maxima in each group. Time zero marks the onset of the presentation of babbling noise, and the dashed line marks the onset of the syllable $600 \mathrm{~ms}$ after the onset of the babbling noise in (b) and (c) trials.

b.
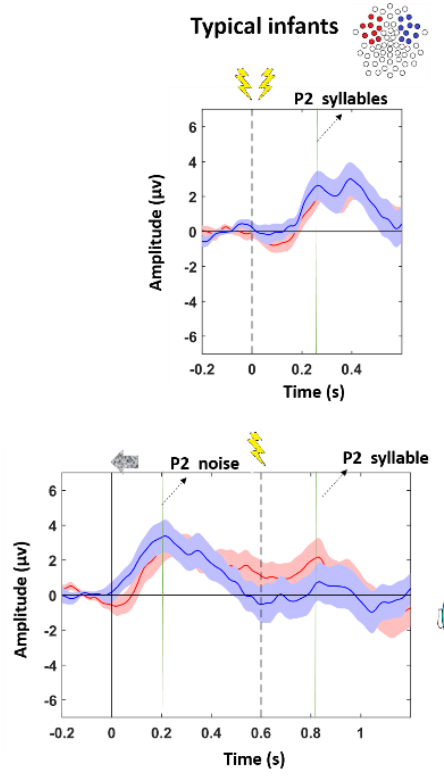

c.

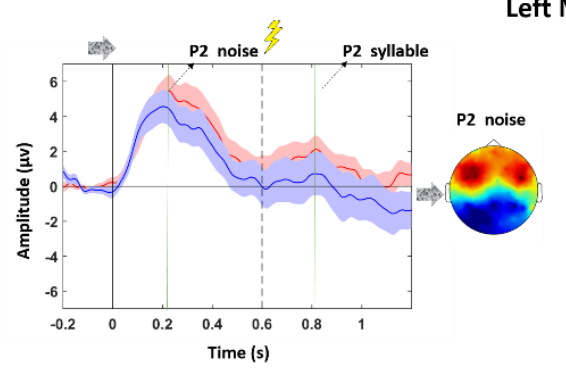

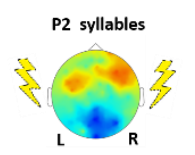
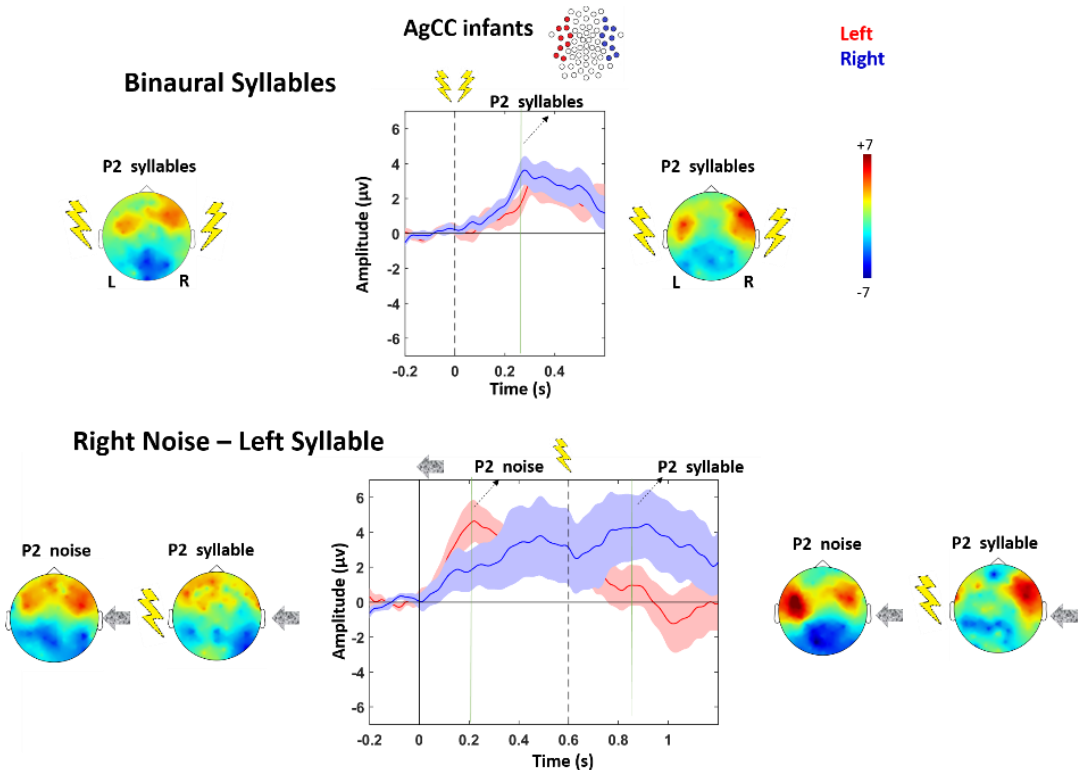

eft Noise - Right Syllable

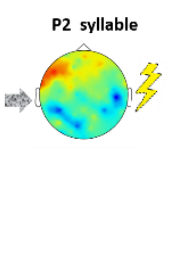

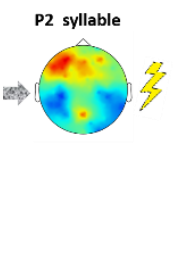


Supplementary figure 2. P2 response amplitude comparison between typical and AgCC infants:

Box plots represent the amplitude of the P2 response measured in typical (black) and AgCC (magenta) infants following stimulation by a) binaural syllables, b) monaural babbling noise (contralateral and ipsilateral responses are shown), and c) dichotic babbling noise-syllable (contralateral and ipsilateral responses are shown in reference to monaural syllable).

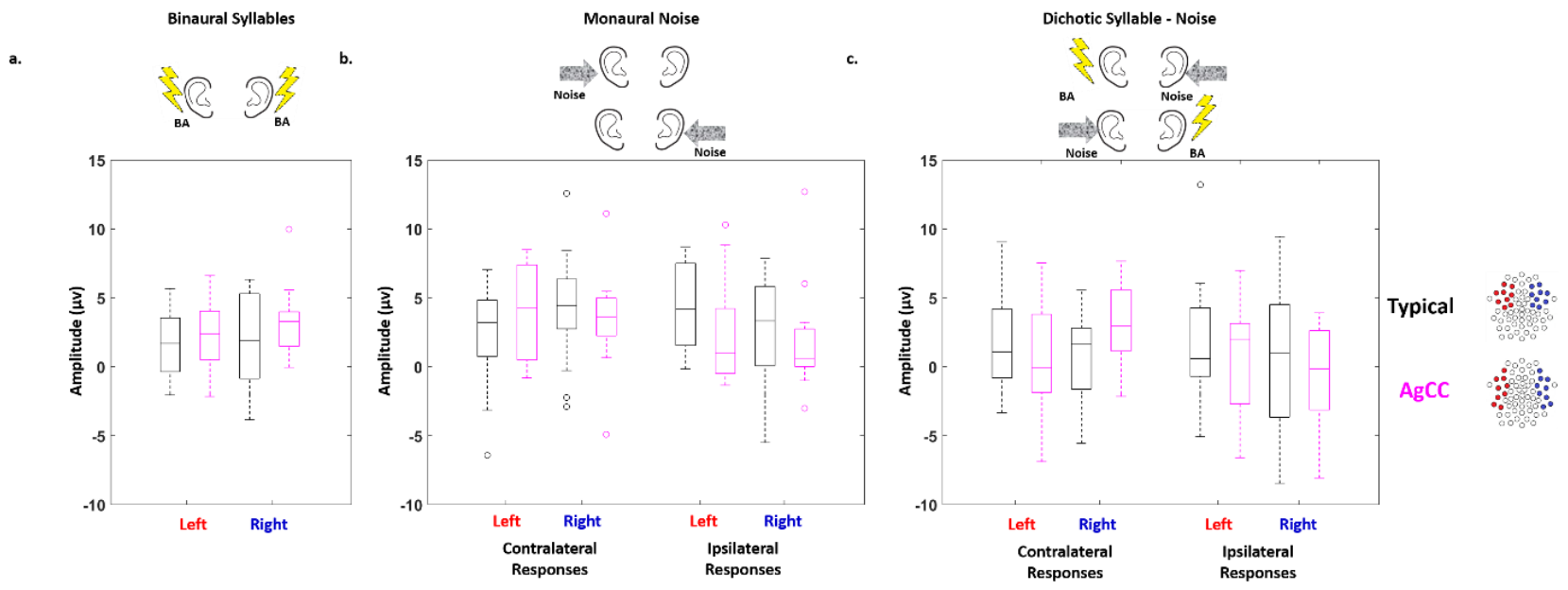

Supplementary figure 3: Influence of the paradigm on the P2 response amplitude:

Box plots represent P2 amplitudes averaged over both hemispheres (left and right sets of electrodes, corresponding to both contralateral and ipsilateral responses) in response to binaural syllables, monaural babbling noise, and dichotic babbling noise-syllables in typical (black) and $\mathrm{AgCC}$ (magenta) infants. Differences between response amplitudes are highlighted with asterisks $(* * \mathrm{p}<0.005 ; * \mathrm{p}<0.5)$, showing that responses for binaural syllables are slower than for other stimuli.

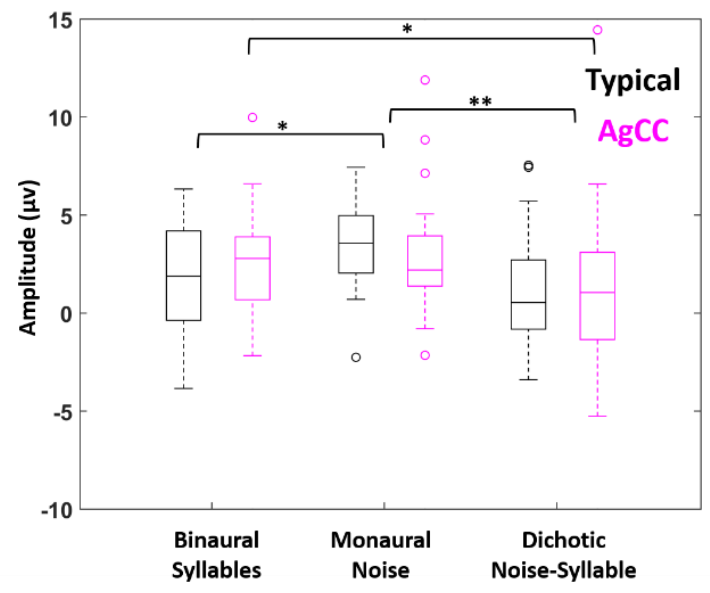


Supplementary figure 4: Topographical differences between typical and AgCC infants:

ERP time courses of the grand average responses in typical (black) and $\mathrm{AgCC}$ (magenta) infants with complete callosal agenesis (excluding the 3 infants with partial $\mathrm{AgCC}$ ) for a) binaural trials; b) dichotic trials: right-ear babbling noise / left-ear syllable trials; c) dichotic trials: left-ear babbling noise / right-ear syllable. In a) and b), similar pattern of between groups differences are observed for the outlined clusters and over the time-windows shaded in light blue or filled with diagonal stripes. Note that the significance of p-values has dropped when $3(\sim 25 \%)$ of the AgCC infants were excluded from the analyses. In c), no group difference was observed, thus we highlighted symmetrical outlined clusters compared to b) (n.s.: non-significant). 2D voltage topographies correspond to the shaded time-windows filled with diagonal stripes.

Binaural Syllables

a.

b.
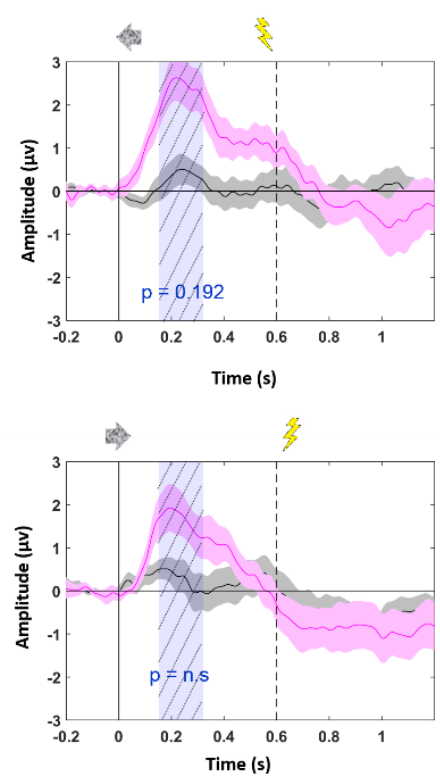
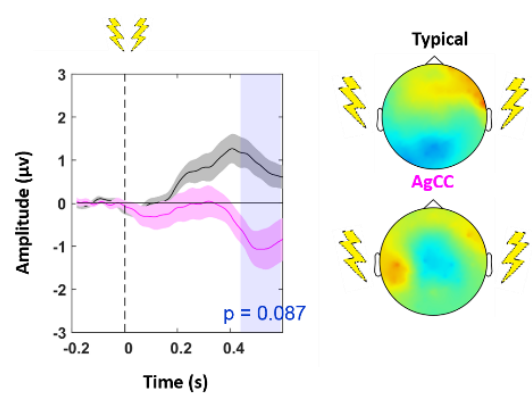

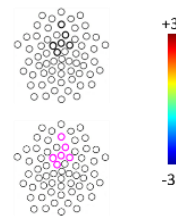

Right Noise - Left Syllable
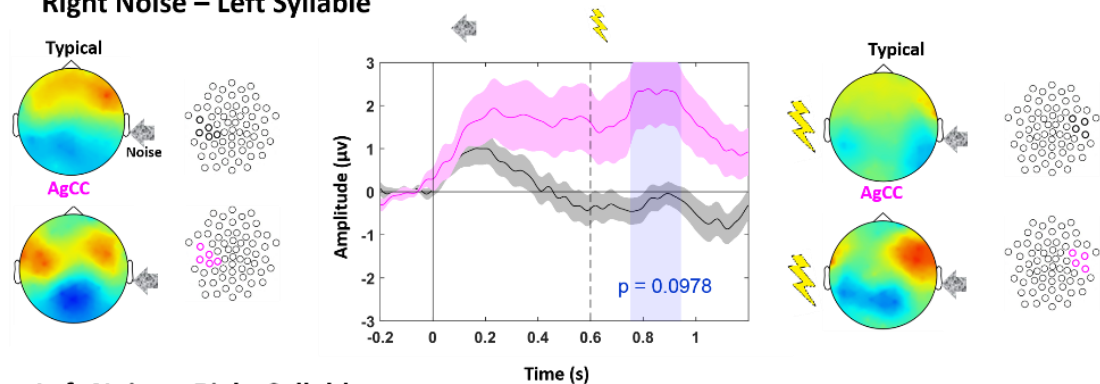

Left Noise - Right Syllable

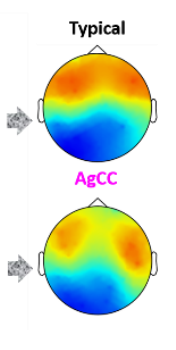

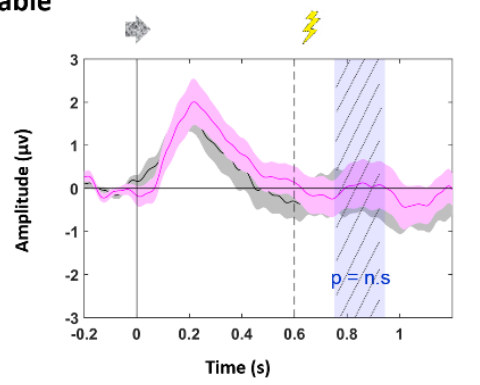

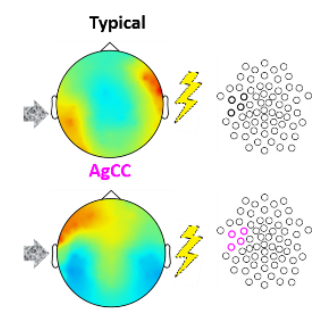


Supplementary table 1: Comparison of the P2 response amplitude between typical and AgCC infants:

The P2 response amplitude was compared between the two groups for different auditory stimuli. The main effects and their interactions are reported.

\begin{tabular}{|c|c|c|}
\hline Binaural & Monaural & Dichotic \\
\hline group $: F(1,29)=1.8, p=0.188$ & group $: F(1,29)=0.9, p=0.335$ & group $: F(1,29)<0.1, p=0.807$ \\
\hline hemisphere $: F(1,29)=1.1, p=0.296$ & hemisphere $: F(1,29)=0.2, p=0.684$ & hemisphere $: F(1,29)<0.1, p=0.780$ \\
\hline group $\times$ hemisphere $: F(1,29)<0.1, p=0.889$ & response side $: F(1,29)=0.3, p=0.599$ & response side $: F(1,29)=1.2, p=0.278$ \\
\hline & group $\times$ hemisphere $: F(1,29)=0.1, p=0.701$ & group $\times$ hemisphere $: F(1,29)=2.0, p=0.165$ \\
\cline { 2 - 3 } & group $\times$ response $\operatorname{side}: F(1,29)=2.5, p=0.122$ & group $\times$ response $\operatorname{side}: F(1,29)=1.8, p=0.183$ \\
\cline { 2 - 3 } & hemisphere $\times$ response side $: F(1,29)=2.2, p=0.146$ & hemisphere $\times$ response side $: F(1,29)=1.7, p=0.195$ \\
\cline { 2 - 3 } & group $\times$ hemisphere $\times$ response $\operatorname{side}: F(1,29)=1.4, p=0.241$ & group $\times$ hemisphere $\times$ response side $: F(1,29)=3.7, p=0.062$ \\
\hline
\end{tabular}

Supplementary table 2: Summary of ANOVA analyses on P2 amplitude in function of the type of auditory stimulation:

The main effects and their interactions are first reported, followed by post-hoc analyses using ttests. Effect sizes (Cohen's d) and 95\% confidence interval (CI) are indicated. Significant pvalues, corrected for multiple comparisons are highlighted by asterisks: $* * \mathrm{p}<0.005 ; * \mathrm{p}<0.5$. bin:binaural; mon:monaural.

\begin{tabular}{|c|}
\hline Dependency of P2 amplitudes on auditory stimulation \\
\hline stimuli type: $F(1,29)=12.5 p<0.001 * *$ \\
\hline group: $F(1,29)<0.1, p=0.797$ \\
\hline hemisphere: $F(1,29)<0.1, p=0.869$ \\
\hline stimuli type $\times$ group: $F(1,29)=1.7, p=0.188$ \\
\hline stimuli type $\times$ hemisphere: $F(2,58)=0.58, p=0.453$ \\
\hline group $\times$ hemisphere $: \mathrm{F}(1,29)=0.6, p=0.454$ \\
\hline stimuli type $\times$ group $\times$ hemisphere $: F(2,58)=1.6, p=0.210$ \\
\hline bin syllable $>$ dichotic syllable:t( $(1,61)=2.2, p=0.032^{*} ;$ \\
d: $0.3,95 \% \mathrm{Cl}:[0.1,2.1]$ \\
mon babbling $>$ dichotic syllable: $\mathrm{t}(1,61)=5, p<0.001^{* *} ;$ \\
d: $0.6,95 \% \mathrm{Cl}:[1.3,3.0]$ \\
mon babbling $>$ bin syllable: $\mathrm{t}(1,61)=2.6, p=0.017^{*} ;$ \\
d: $0.3,95 \% \mathrm{Cl}:[0.2,1.9]$
\end{tabular}

Supplementary table 3: ANOVAs of P2 response latency in the binaural, monaural and dichotic trials when excluding the 3 infants with partial AgCC from the AgCC group:

The P2 response latency was compared between the two groups for different auditory stimuli. For each ANOVA, the main effects and their interactions are first reported, followed by post-hoc analyses using t-tests when interactions were significant. Effect sizes (Cohen's d) and 95\% confidence intervals (CI) on the averaged differences are indicated. Significant p-values, corrected for multiple comparisons are highlighted by asterisks: $* * \mathrm{p}<0.005 ; * \mathrm{p}<0.5$. AgCC: agenesis of corpus callosum, ipsi: ipsilateral, contra: contralateral, L: left hemisphere, R: right hemisphere. 


\begin{tabular}{|c|c|c|}
\hline Binaural & Monaural & Dichotic \\
\hline group : $F(1,26)=3.6, p=0.067$ & group : $F(1,26)<0.1, p=0.946$ & group : $F(1,26)=0.6, p=0.427$ \\
\hline \multirow[t]{2}{*}{ hemisphere: $F(1,26)<0.1, p=0.889$} & hemisphere : $F(1,26)=26.4, p<0.001 * *$ & hemisphere: $F(1,26)<0.1, p=0.879$ \\
\hline & response side : $F(1,26)=0.4, p=0.536$ & response side $: F(1,26)=1.6, p=0.214$ \\
\hline \multirow{5}{*}{ group $x$ hemisphere $: F(1,26)=0.8, p=0.371$} & group $\times$ hemisphere : $F(1,26)=2.6, p=0.121$ & group $x$ hemisphere $: F(1,26)<0.1, p=0.764$ \\
\hline & group $\times$ response side $: F(1,26)=4.4, p=0.045^{*}$ & group $\times$ response side $: F(1,26)=0.3, p=0.584$ \\
\hline & hemisphere $x$ response side $: F(1,26)=3.5, p=0.072$ & hemisphere $x$ response side : $F(1,26)=2.3, p=0.144$ \\
\hline & group $\mathrm{x}$ hemisphere $\mathrm{x}$ response side $\mathrm{F}(1,26)=7.2, \mathrm{p}=0.013 *$ & group $x$ hemisphere $x$ response side $: F(1,26)=0.5, p=0.489$ \\
\hline & 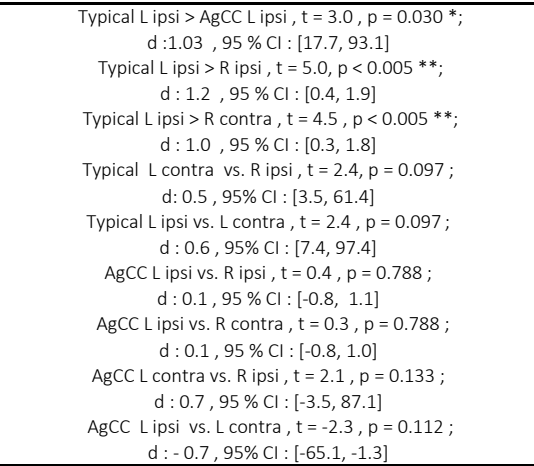 & \\
\hline
\end{tabular}

Supplementary table 4: ANOVAs of P2 response amplitude in the binaural, monaural and dichotic trials when excluding the 3 infants with partial AgCC from the AgCC group:

The P2 response amplitude was compared between the two groups for different auditory stimuli. For each ANOVA, the main effects and their interactions are reported.

\begin{tabular}{|c|c|c|}
\hline Binaural & Monaural & Dichotic \\
\hline group $: F(1,26)=0.8, p=0.369$ & group $: F(1,26)=0.8, p=0.372$ & group $: F(1,26)=0.2, p=0.594$ \\
\hline hemisphere $: F(1,26)=1.0, p=0.323$ & hemisphere $: F(1,26)<0.1, p=0.932$ & hemisphere $: F(1,26)=0.1, p=0.740$ \\
\hline group $\times$ hemisphere $: F(1,26)<0.1, p=0.846$ & response side $: F(1,26)<0.1, p=0.876$ & response side $: F(1,26)=1.1, p=0.303$ \\
\hline & group $\times$ hemisphere $: F(1,26)<0.1, p=0.826$ & group $\times$ hemisphere $: F(1,26)=1.9, p=0.178$ \\
\cline { 2 - 3 } & group $\times$ response side $: F(1,26)=0.7, p=0.401$ & group $\times$ response side $: F(1,26)=2.2, p=0.152$ \\
\cline { 2 - 3 } & hemisphere $\times$ response $\operatorname{side}: F(1,26)=2.4, p=0.132$ & hemisphere $\times$ response side $: F(1,26)=0.9, p=0.348$ \\
\hline
\end{tabular}

